Original Research Article

\title{
A randomized open label comparative study to determine the various side effects and patient satisfaction of low dose continuous versus low dose intermittent oral isotretinoin therapy in moderate to severe acne vulgaris
}

\author{
Santoshkumar A. Shetti ${ }^{1}$, Nagesh H. N. ${ }^{2 *}$, Nagabushan Hanumanthaiah ${ }^{3}$
}

\begin{abstract}
${ }^{1}$ Department of Pharmacology, Bharati Vidyapeeth (Deemed to be University) Medical College and Hospital, Sangli,

Maharashtra, India

${ }^{2}$ Department of Pharmacology, Akash Institute of Medical Sciences and Research Centre, Bengaluru, Karnataka, India ${ }^{3}$ Department of Pharmacology, Mandya Institute of Medical Sciences, Mandya, Karnataka, India
\end{abstract}

Received: 27 March 2018

Revised: 04 April 2018

Accepted: 25 April 2018

*Correspondence to:

Dr. Nagesh H. N.,

Email: nagu728@gmail.com

Copyright: () the author(s), publisher and licensee Medip Academy. This is an openaccess article distributed under the terms of the Creative Commons Attribution NonCommercial License, which permits unrestricted noncommercial use, distribution, and reproduction in any medium, provided the original work is properly cited.

\begin{abstract}
Background: Acne Vulgaris is chronic inflammatory disease of pilosebaceous units. Oral isotretinoin is recommended for moderate to severe acne vulgaris who are not responding satisfactorily to conventional therapies. Recent reports indicate that acne patients have been benefiting from the low dose treatment protocols. However, long term daily use of this drug results in frequent side effects such mucocutaneous and systemic side effects. Our aim was to assess and compare the various side effects and patient satisfaction of oral isotretinoin in low dose continuous and intermittent treatment of moderate to severe acne vulgaris. Methods: This was a prospective randomized open labeled comparative study carried out at outpatient department in the Department of Dermatology in Mandya Institute of Medical sciences, Mandya. Patients with moderate to severe acne were assigned equally ( 50 subjects each) to one of the two treatment regimens by using block randomization technique, Group A was given low dose continuous regimen-20 mg oral isotretinoin once daily for 4 months and Group B was given low dose intermittent regimen-20 mg oral isotretinoin once daily for 1 week out of every 4 weeks. The patients were followed up every 4 th week during the treatment period. The patients were examined and side effects were noted in each visit. A six month follow-up evaluation was done to analyze patient satisfaction. Results: Muco-cutaneous dryness was most common adverse effect noted in both the groups A and B. Itching (42\%), Alopecia (44\%), Myalgia (36\%) were seen most commonly in group A and Acne flaring (47\%) was most common with group B. With regard to patient satisfaction, in group A $42 \%$ were satisfied and $20 \%$ were very satisfied, in group B $36 \%$ were satisfied and $14 \%$ were very satisfied.

Conclusions: Study suggests that, Muco-cutaneous dryness was most common side effect in both treatment regimens. Side effects were more frequent with low dose continuous than low dose intermittent isotretinoin regimen. Patient satisfaction was better in continuous regimen.
\end{abstract}

Keywords: Acne vulgaris, Conventional therapy, Oral isotretinoin, Mucocutaneous side effects

\section{INTRODUCTION}

Acne Vulgaris is chronic inflammatory disease of pilosebaceous units characterized by comedones, papules, pustules, nodules, cysts, abscesses, and later on sometimes as widespread scarring. This disease occurs worldwide and usually starts in adolescence and resolves by midtwenties. ${ }^{1}$ It is the most common skin disorder and, 
prevalence of moderate to severe acne vulgaris being about $11 \% .^{2}$

According to the severity of acne there are various modalities of treatment and they include both systemic and topical therapy. Systemic therapy includes systemic antibiotics, hormonal therapy and oral isotretinoin. The topical treatment includes Benzoyl peroxide (2.5-10\%), Topical retinoids (tretinoin, isotretinoin, adapalene, tazarotene etc.), Topical antibiotics (erythromycin, clindamycin etc.), and other topical agents like (salicylic acid, azelaic acid etc.). ${ }^{3}$

Oral isotretinoin is recommended for moderate to severe acne vulgaris who are not responding satisfactorily to conventional therapies. Recent reports indicate that acne patients have been benefiting from the low dose treatment protocols. However, long term daily use of this drug results in frequent side effects, some of which may lead to disastrous complications resulting in difficulties in complying with the treatment. ${ }^{4}$ Oral retinoids have multiple mucocutaneous and systemic side effects. Mucocutaneous toxicity is the most commonly observed side effect of isotretinoin use. This study to determine and compare the various side effects with oral isotretinoin in low dose continuous and intermittent treatment of moderate to severe acne vulgaris.

\section{Objectives}

- Primary objective: To determine the various side effects and patient satisfaction with oral isotretinoin in low dose continuous and intermittent treatment of moderate to severe acne vulgaris.

- Secondary objectives: To compare the various side effects and patient satisfaction with oral isotretinoin in low dose continuous and intermittent treatment of moderate to severe acne vulgaris

\section{METHODS}

After institutional ethical committee approval, a randomized open label study was conducted from July 2012 to August 2013 in patients attending the outpatient clinic in the Department of Dermatology in Mandya Institute of Medical Sciences, Mandya. The study was approved by the ethics committee of Mandya Institute of Medical Sciences.

Prospective randomized (Block randomization) open label study.

\section{Sample size calculation ${ }^{5}$}

$$
\begin{gathered}
\text { Sample size }(\mathrm{n})=\mathrm{Z}^{2} \mathrm{P}(1-\mathrm{P}) / \mathrm{d}^{2}=1.96^{2} \mathrm{X} 96.03(1- \\
96.03) /(19.18)^{2}=\sim 95
\end{gathered}
$$

( $\mathrm{Z}$ is 1.96 for $95 \%$ Confidence interval, $\mathrm{P}=$ maximum response score and $\mathrm{d}=$ the difference between maximum and minimum score)

\section{Inclusion criteria}

The patients those who volunteered to give informed consent, male and female patients in the age range of 1845 years and diagnosed to have moderate to severe acne, willing to take oral isotretinoin therapy and not responded to antibiotic therapy.

\section{Exclusion criteria}

Patients with Diabetes mellitus, Allergy to isotretinoin drugs, on oral contraceptives and other drugs known to produce acne, Pregnant and breast-feeding women, subjects with abnormal lipid profile, significant hepatic dysfunction and underlying psychiatric disorders.

The subjects were explained in the language best understood by them about the purpose of study and its benefits to them as well as possible adverse effects. After obtaining written informed consent patients sociodemographic profile with family history of acne were taken. Based on the Global Acne Grading scale (Table 1) out of 126 patients screened 100 subjects were diagnosed to have moderate to severe acne. 50 subjects in each group were randomly assigned by using block randomization technique: Group A received low dose continuous regimen - 20mg oral isotretinoin once daily for 4 months and Group $B$ received low dose intermittent regimen - 20mg oral isotretinoin once daily for 1 week out of every 4 weeks. The patients were followed up every 4th week during the treatment period. A six month follow-up evaluation after the end of treatment was performed to analyze patient satisfaction.

The patients were examined, and side effects were noted in each visit.

Table 1: Global acne grading scale (for patient selection).

\begin{tabular}{|ll|}
\hline Age group & Parameter \\
\hline 0 & None \\
\hline $1-18$ & Mild \\
\hline $19-30$ & Moderate \\
\hline $31-38$ & Severe \\
\hline$\geq 39$ & Very severe \\
\hline
\end{tabular}

At the end of the study, the degree of satisfaction on a fourpoint scale (Table 2) was documented by the participants.

Table 2: Degree of satisfaction on a four point scale.

\begin{tabular}{|ll|}
\hline Four point scale & Parameter \\
\hline 4 & Very satisfied \\
\hline 3 & Satisfied \\
\hline 2 & Slightly satisfied \\
\hline 1 & Dissatisfied \\
\hline
\end{tabular}


Data was entered into Microsoft excel and analyses were done using the Statistical Package for Social Sciences (SPSS). Descriptive statistics such as mean and standard deviation (SD) for continuous variables, and frequency and percentage for categorical variables were determined. Unpaired ' $t$ ' test was used to compare means between group A and group B for continuous variables. $\mathrm{P}<0.05$ was considered as significant.

\section{RESULTS}

In the study, a total of 100 patients were recruited, 50 patients were received continuous low dose oral isotretinoin regimen (group A) and another 50 were received intermittent low dose oral isotretinoin regimen (group B).

There were $72 \%$ males and $28 \%$ were females. The prevalence age was ranged from $20-22$ years. $58 \%$ of the patients had Papulo-pustular and $40 \%$ had pustule- nodular lesions in group A and $88 \%$ of patients in group B had maculopapular lesion. $96 \%$ of study subjects in group A showed severe form of acne and 94\% in group B showed moderate form of acne (Table 3 ).

Table 3: Bio-social characteristics in two groups $(\mathbf{N}=\mathbf{1 0 0})$.

\begin{tabular}{|c|c|c|}
\hline $\begin{array}{l}\text { Bio-Social } \\
\text { characteristics }\end{array}$ & $\begin{array}{l}\text { Group A } \\
(\mathrm{n}=50)\end{array}$ & $\begin{array}{l}\text { Group B } \\
(\mathrm{n}=50)\end{array}$ \\
\hline \multicolumn{3}{|l|}{ Age (in years) } \\
\hline Mean \pm SD & $21.74 \pm 2.07$ & $21.02 \pm 2.29$ \\
\hline \multicolumn{3}{|l|}{ Sex } \\
\hline Male & $35(70 \%)$ & $37(74 \%)$ \\
\hline Female & $15(30 \%)$ & $13(26 \%)$ \\
\hline \multicolumn{3}{|l|}{ Weight (in kg) } \\
\hline Mean \pm SD & $56.1 \pm 5.12$ & $56.3 \pm 5.26$ \\
\hline \multicolumn{3}{|l|}{ Marital status } \\
\hline Unmarried & $50(100 \%)$ & $50(100 \%)$ \\
\hline Married & 0 & 0 \\
\hline \multicolumn{3}{|l|}{ Family history } \\
\hline Present & $18(36 \%)$ & $12(24 \%)$ \\
\hline Absent & $32(64 \%)$ & $38(76 \%)$ \\
\hline \multicolumn{3}{|l|}{ Type of Acne } \\
\hline Maculo-papular lesions & $1(2 \%)$ & $44(88 \%)$ \\
\hline Papulo-pustular & $29(58 \%)$ & $6(12 \%)$ \\
\hline Pustule-nodular & $20(40 \%)$ & 0 \\
\hline \multicolumn{3}{|l|}{ Grading of acne } \\
\hline Moderate & $2(4 \%)$ & $47(94 \%)$ \\
\hline Severe & $48(96 \%)$ & $3(6 \%)$ \\
\hline
\end{tabular}

In the study subjects commonly seen adverse effects were mucocutaneous dryness $(99 \%)$, itching $(60 \%)$, acne flare (54\%), alopecia (54\%) and myalgia (42\%) (Figure 1).

Muco-cutaneous dryness (Figure 2) was most common adverse effect noted in both the groups A and group B. Itching (42\%), Alopecia (44\%), Myalgia (36\%) were seen most commonly in group A and Acne flaring (47\%) was most common with group B and there was statistical difference $(\mathrm{P}<0.001)$ between the groups. Acne flaring (14\%) was least common side effect in group A (Table 4 and Figure 3).

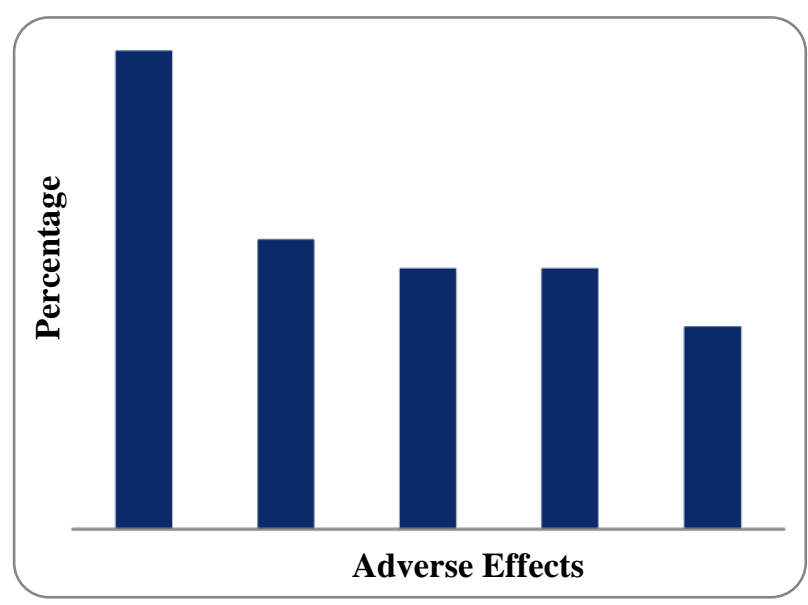

Figure 1: Distribution of patients according to their adverse effects during therapy for acne vulgaris $(\mathbf{N}=\mathbf{1 0 0})$.

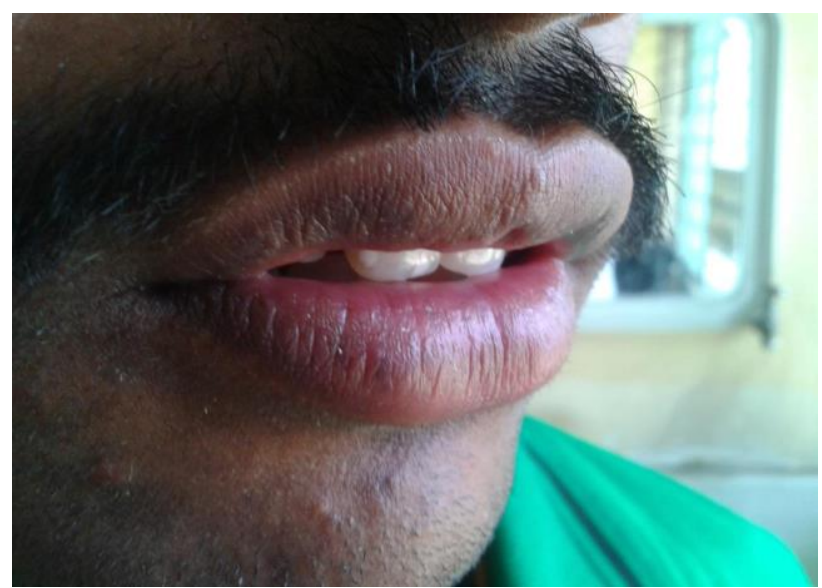

Figure 2: Mucocutaneous side effects.

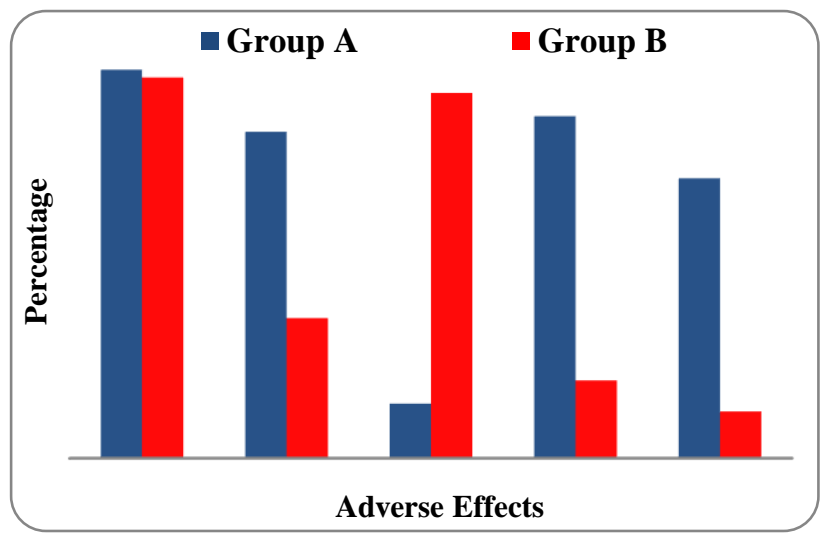

Figure 3: Association of adverse effects seen in Group $A$ and Group B in the low dose oral isotretinoin treatment of acne vulgaris patients. 
Table 4: Association of mode of therapy for acne vulgaris patients with their adverse effects during their therapy $(\mathbf{N}=100)$ bio-social characteristics.

\begin{tabular}{|c|c|c|c|}
\hline & $\begin{array}{l}\text { Group A } \\
(\mathbf{n}=\mathbf{5 0})\end{array}$ & $\begin{array}{l}\text { Group B } \\
(n=50)\end{array}$ & P Value \\
\hline \multicolumn{4}{|c|}{ Muco-cutaneous dryness } \\
\hline Yes & $50(100.0)$ & $49(98.0)$ & \multirow{2}{*}{0.315} \\
\hline No & $0(0.0)$ & $1(2.0)$ & \\
\hline \multicolumn{4}{|c|}{ Itching } \\
\hline Yes & $42(84.0)$ & $18(36.0)$ & \multirow{2}{*}{$<0.001$} \\
\hline No & $8(16.0)$ & $32(64.0)$ & \\
\hline \multicolumn{4}{|c|}{ Acne flare } \\
\hline Yes & $7(14.0)$ & $47(94.0)$ & \multirow{2}{*}{$<0.001$} \\
\hline No & $43(86.0)$ & $3(6.0)$ & \\
\hline \multicolumn{4}{|c|}{ Alopecia } \\
\hline Yes & $44(88.0)$ & $10(20.0)$ & \multirow{2}{*}{$<0.001$} \\
\hline No & $6(12.0)$ & $40(80.0)$ & \\
\hline \multicolumn{4}{|c|}{ Myalgia } \\
\hline Yes & $36(72.0)$ & $6(12.0)$ & \multirow{2}{*}{$<0.001$} \\
\hline No & $14(28.0)$ & $44(88.0)$ & \\
\hline
\end{tabular}

Statistical significant difference seen in the side effects of itching, acne flaring, alopecia and myalgia between Group A and Group B $(\mathrm{p}<0.001)$

With regard to patient satisfaction, in group A $42 \%$ were satisfied and 20\% were very satisfied, in group B $36 \%$ were satisfied and $14 \%$ were very satisfied (Figure 4 ).

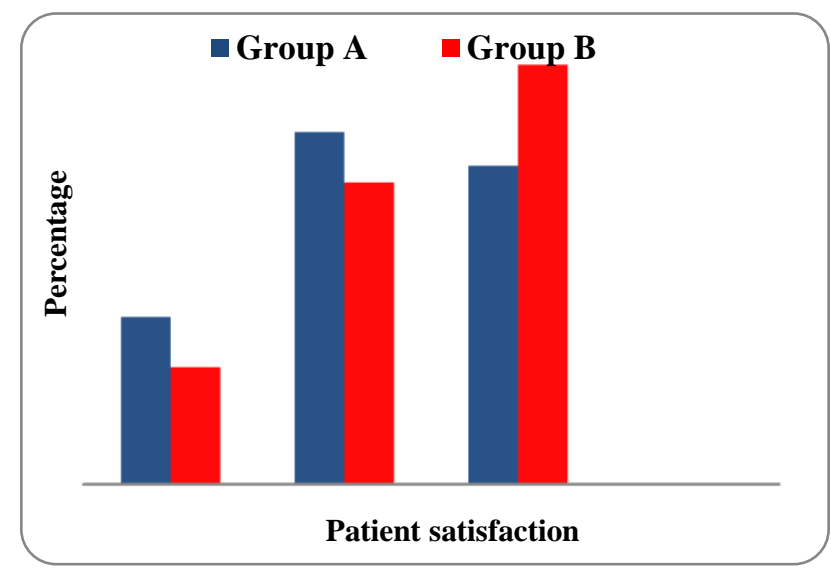

Figure 4: Patient satisfaction with low dose oral isotretinoin therapy in continuous (Group A) and intermittent (Group B) regimen.

\section{DISCUSSION}

Acne vulgaris is a chronic, inflammatory disease of pilosebaceous units, characterized by comedones, papules, pustules, nodules and often scars. Many factors including androgenic stimulation, propionibacterium acnes activity, sebum production, hypercornification, as well as inflammatory mediator responses are thought to play a role in acne pathogenesis.
Isotretinoin is the only drug that affects almost all factors in acne pathogenesis and is now established as a successful therapeutic option with ability to induce long term remission in patients with acne vulgaris. Isotretinoin was earlier prescribed for cases of nodulocystic acne but is now increasingly used to treat patients of moderate to severe acne vulgaris, which are not responsive to topical therapy or oral antibiotics. ${ }^{2,6}$

In the study muco-cutaneous dryness was most common adverse effect noted in both the groups, similar result was observed in Lee JW et al. ${ }^{2}$ study but Rao PK et al, study showed cheilitis was the most common among the side effects observed and was seen in $98 \%$ of the participants. ${ }^{7}$

Nadia A El-Sherif et al, study showed no statistically significant differences among both groups regarding the frequency and severity of the side effects. ${ }^{8}$ In this study the frequency and severity of treatment related side effects like dry skin, alopecia, itching was significantly higher in low dose continuous regimen as compared to low dose intermittent regimen. Acne flaring was more common in low dose intermittent than low dose continuous.

The patient satisfaction score was highest in low dose continuous regimen as compared with intermittent regimen. This result suggests that the low dose continuous regimen is slightly superior to low dose intermittent regimen in terms of patient satisfaction. Boyraz, $\mathrm{N}$ et al, and Lee JW et al, studies had shown similar results. ${ }^{2,9}$

The result of our study suggests that, low dose continuous regimen has shown higher adverse effect and better patient satisfaction than low dose intermittent regimen for patients with moderate to severe acne.

\section{Limitations}

The sample size was small. Not many investigations done to detect side effects.

\section{CONCLUSION}

The result of our study suggests that, low dose continuous regimen has shown higher adverse effect and better patient satisfaction than low dose intermittent regimen for patients with moderate to severe acne. Muco-cutaneous dryness was most common side effect in both treatment regimens. Itching, alopecia and myalgia were common with low dose continuous and acne flaring was most commonly seen in intermittent regimen.

\section{ACKNOWLEDGEMENTS}

Authors would like to thank Dr. Harish M R., Professor and Head, Dr. Shashikumar N, Associate professor, Department of Dermatology, Mandya Institute of Medical Sciences, Mandya for their constant support, guidance and providing resources for this study. 
Funding: No funding sources

Conflict of interest: None declared

Ethical approval: The study was approved by the

Institutional Ethics Committee

\section{REFERENCES}

1. Dreno B, Poli F. Epidemiology of acne. Dermatology. 2003;43:1042-8.

2. Lee JW, Yoo KH, Park KY, Han TY, Li K, Seo SJ, et al. Effectiveness of conventional, low dose and intermittent oral isotretinoin in the treatment of acne: a randomized, controlled comparative study. $\mathrm{J} \mathrm{Br}$ Dermatol. 2011;164:1369-75.

3. Rathi SK. Acne vulgaris treatment: The Current Scenario. Indian J Dermatol Venereol. 2011;56:7-13.

4. Akman A, Durusoy C, Senturk M, Koc CK, Soyturk D, Alpsoy E. Treatment of acne with intermittent and conventional isotretinoin: a randomized, controlled multicenter study. Arch Dermatol Res. 2007;299:46773.

5. Agarwal US, Besarwal RK, Bhola K. Oral isotretinoin in different dose regimens for acne vulgaris: A randomized comparative trial. Indian J Dermatol Venerol Leprol. 2011;77:688-94.
6. Melnik BC. FoxO1- the key for the pathogenesis and therapy of acne? J Dtsch Dermatol Ges. 2010;8:10514.

7. Rao PK, Bhat RM, Nandakishore B, Dandakeri S, Martis J, Kamath GH. Safety and Efficacy of LowDose Isotretinoin in the Treatment of Moderate to Severe Acne Vulgaris. Ind $J$ of Dermatol. 2014;59(3):316.

8. Nadia A El-Sherif, Azza SH Greiw, Benamer AM. Efficacy of low dose versus intermittent isotretinoin regimens in patients with moderate acne vulgaris: A randomized controlled trial. Ibnosina J Med B S. 2013;5(5):296-302.

9. Boyraz N, Mustak PK. Comparison of the efficacies of intermittent and continuous low-dose isotretinoin regimens in the treatment of moderate acne vulgaris. Int J of Dermatol. 2013 Oct 1;52(10):1265-7.

Cite this article as: Shetti SA, Nagesh HN, Hanumanthaiah N. A randomized open label comparative study to determine the various side effects and patient satisfaction of low dose continuous versus low dose intermittent oral isotretinoin therapy in moderate to severe acne vulgaris. Int J Basic Clin Pharmacol 2018;7:1169-73. 